\title{
CAM in the Production of Casting Patterns
}

Ondřej Bílek MSc., Ph.D., Luboš Rokyta MSc., Jaroslav Šimoník MSc.

Tomas Bata University in Zlin, Department of Production Engineering, T.G. Masaryka 5555, 76001 Zlín, Czech Republic.bilek@ft.utb.cz

The article is focused on the production of permanent casting patterns from wood for impermanent moulds by the 5-axis machining centre. Programming of casting pattern was dealt with CAM software by two different manufacturers. Casting pattern, adapted by appropriate allowances, was loaded in the SolidCAM R10 and the CAM Express 7.5 by Siemens. Ergonomics in the working environment of programmes, operation verifications and theoretical machine time were compared. The casting pattern was machined after generating the NC code on FC3800 MACH milling machine. Monitored parameters during computer programming were associated with the measured values when machining casting pattern. Estimated parameters between the computer simulations differed minimally in general, although the programming methods of cutting operations were different. Considerable differences, as expected, were observed in the values of estimated and actual machine time.

Key words: CAM, CNC Milling, Casting Pattern, Software.

\section{References}

[1] BÍLEK, O., ROKYTA, L. (2011) Rapid Prototyping in Casting Technology: Case Study. Annals \& Proceedings of 22nd DAAAM World Symposiums. ISBN 978-3-901509-83-4. (In press).

[2] HRDINA, J. Zkoušky trvanlivosti povlakovaných nástrojů. Vrstvy a povlaky 2008 - Zborník prednášok. Rožnov pod Radhoštěm, 29.-30.9.2008. Trenčín : DIGITAL GRAPHIC, 2008, p. 68-73. ISBN 978-80-969310-7-1.

[3] JANDEČKA, K. Postprocesory a programování NC strojů. Ústí nad Labem, 2007.

[4] JANDEČKA, K., ČESÁNEK, J., ŠKARDA, J. (2006) Postprocessor of CAD/CAM System Cimatron and New Types of Interpolation. Manufacturing Technology, Vol. 1, No. VI. Pp. 34-40. ISSN 1213248-9.

[5] JANDEČKA, K., SKOPEČEK, T. (2004). Optimalizace řezného procesu a moderní CAD/CAM systémy. Strojírenská technologie. Vol. 1, No. IX, pp. 15-19. ISSN 12114162.

[6] JURKO, J., LUKOVICS, I. Obrábatel’nost’ materiálov. 1.vyd, Zlín: UTB, 2008. 144p. ISBN 978-80-7318-7361.

[7] MÁDL, J., HOLEŠOVSKÝ, F., NOVÁK, M. Strojírenská technologie pro moderní výrobu. FVT UJEP : Ústí n. Labem. 2010. 56s. ISBN 978-80-7414-218-5.

[8] MAREK, J. Konstrukce CNC obráběcích strojů. Praha: MM publishing, 2010. 420 s. ISBN 978-80-254-7980-3.

[9] NOVÁK, M. (2010) Možnosti hodnocení kvality obrobených povrchů. In: Strojírenská technologie. zvl. Vydání. Ústí n. Labem : FVTM UJEP, 2010. s. 195-198. ISSN 1211-4162.

[10] RAO, R. N. CAD/CAM: principles and applications. Tata McGraw-Hill Publishing Company Limited. 2006, 253 p., ISBN 0-07-058373-0.

[11] SMID, P. CNC programming handbook : a comprehensive guide to practical CNC programming. 2003. New York : Industrial Press Inc, 2003. 508 s. ISBN 0-8311-3158-6.

[12] VASILKO, K. (2010). The wood requires orthogonal cutting. Manufacturing Technology, Vol. X., No. 10. December 2010. pp 39-45, ISSN 1213-2489.

Paper number: M201202

Manuscript of the paper recieved in 2011-11-21. The reviewers of this paper: Assoc. Prof. Dr. Josef Chladil, MSc. and Prof. Karel Jandecka, MSc., Ph.D. 